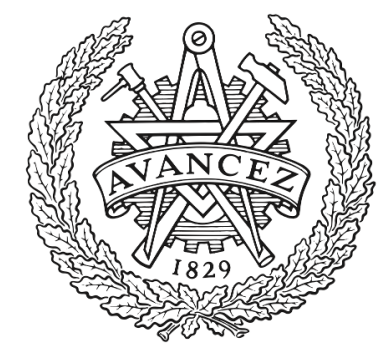

CHALMERS

UNIVERSITY OF TECHNOLOGY

\title{
Theoretical Investigation of Longitudinal Dispersion Fluctuations on All-Fiber Phase-Sensitive Parametric Optical Switch
}

Downloaded from: https://research.chalmers.se, 2023-04-26 07:17 UTC

Citation for the original published paper (version of record):

Gao, J., Meng, Y., Fu, S. et al (2017). Theoretical Investigation of Longitudinal Dispersion Fluctuations on All-Fiber Phase-Sensitive

Parametric Optical Switch. Journal of Lightwave Technology, 35(9): 1646-1653.

http://dx.doi.org/10.1109/jlt.2017.2681685

N.B. When citing this work, cite the original published paper. 


\title{
Theoretical Investigation of Longitudinal Dispersion Fluctuations on All-Fiber Phase-Sensitive Parametric Optical Switch
}

\author{
Jitao Gao, Yan Meng, Songnian Fu, Magnus Karlsson, Peter A. Andrekson, \\ Ming Tang, and Deming Liu
}

\begin{abstract}
A fiber-based all-optical switch accomplished by pump depletion in a single-pump phase sensitive (PS) amplifier is theoretically investigated using an inhomogeneous fiber model including the zero-dispersion wavelength (ZDW) fluctuations. We find that the OFF-state (maximum pump depletion) of the PS alloptical switch is very sensitive to the ZDW fluctuations, whereas the ON-state (minimum pump depletion) is more robust. Meanwhile, the nonreciprocal transmission characteristic of the PS all-optical switch induced by the ZDW fluctuations is investigated with the help of the proposed inhomogeneous fiber model. Our theoretical results are useful to optimize the experimental implementation of PS all-optical switch.
\end{abstract}

Index Terms-Fiber nonlinear optics, four-wave mixing, optical switches, phase sensitive amplifiers.

\section{INTRODUCTION}

$\mathbf{F}$ IBER optic parametric amplifiers (FOPAs) have been extensively investigated in recent years. In contrast to the conventional optical amplifiers, such as erbium-doped fiber amplifiers (EDFAs) and Raman amplifiers, FOPAs can provide much higher gain over a relatively wide bandwidth [1], [2]. Moreover, FOPAs can be used in many other areas, including all-optical signal regeneration [3], [4], wavelength conversion and multicasting [5]-[7], low-noise optical amplification [8], and wideband optical frequency comb generation [9]. In addition, FOPAs, based on the four-wave mixing (FWM) effect arising in a highly nonlinear fiber (HNLF), can be viewed as a photon-exchange device with ultrafast (femtosecond) response time, where the pump power is transferred to the signal and

This work was supported in part by the National Natural Science Foundation of China under Grant 61575071 and Grant 61711530043, in part by the 863 High Technology Plan (2015AA015502), in part by the European Research Council under Grant Agreement ERC-2011-AdG-291618 PSOPA, and in part by the K. A. Wallenberg Foundation.

J. Gao, Y. Meng, S. Fu, M. Tang, and D. Liu are with the National Engineering Laboratory for Next Generation Internet Access System, School of Optics and Electronic Information, Huazhong University of Science and Technology, Wuhan 430074, China (e-mail: songnian@mail.hust.edu.cn).

M. Karlsson and P. A. Andrekson are with the Photonics Laboratory, Department of Microtechnology and Nanoscience, Chalmers University of Technology, Gothenburg 41296, Sweden. idler wavelengths. In particular, for a gain-saturated FOPA, not only the signal is amplified, but also the pump power is depleted [10]. In previous works, it was demonstrated that in a gain-saturated FOPA even a weak (few-photon) signal was sufficient to deplete a high-power pump. This provides a mechanism of great potential application in ultrafast all-optical switching devices with energy-efficient operation [11]-[13]. Those optical switches were all operated in phase-insensitive (PI) mode. When a pulsed signal is used to control the pulsed pump, walkoff among the involved waves occurs. Especially, in order to maximize the pump depletion, the wavelengths of the pulsed pump and pulsed signal are usually set near the zero-dispersion wavelength (ZDW), which may cause dispersion-induced pulse breakup and severely deteriorate the performance of a PI alloptical switch [12]. Moreover, for the PI optical switch, the pump intensity is controlled by the optical intensity of a weak probe signal, and it is insensitive to the phases of the involving waves. In contrast, the single-pump phase sensitive (PS) FOPA has a higher gain and saturates at lower input signal powers than its PI counterpart [14]-[16]. In addition, the signal and idler waves are either amplified or de-amplified depending on the relative phase of the involved waves in the gain-saturated PS-FOPA, in contrast with the gain-saturated PI-FOPA. Hence, the pump depletion and the output pump power of the gainsaturated PS-FOPA are sensitive to the relative phase. As a result, the pump wave can be switched when the phases of one or two of the signal and idler waves are tuned under the condition of ultra-low power injection. Furthermore, due to the use of continuous-wave (CW) signal and idler, the effects of pulse breakup and walk-off existing in the PI all-optical switch can be significantly mitigated. Therefore, the fiber-based PS all-optical switch might be an attractive and promising all-optical device in future energy-efficient optical networks [17]. However, a FOPA is a distributed optical amplifier and the FWM effect is sensitive to the local phase matching [13]. During the HNLF manufacture, variations of the core diameter are inevitably introduced, which causes ZDW fluctuations along the fiber. Therefore, fiber segments with the same global dispersion parameters (i.e. average ZDW and dispersion slope) may have different ZDW profiles. The first investigation [18] of ZDW fluctuations modeled them as a Gaussian random stochastic process and found that longlength scale fluctuations are much more devastating than the 
short-length ones for the performance of a FOPA. Following investigations were mainly focused on the effects of dispersion fluctuations on the signal gain [19], flat gain bandwidth [20], noise characteristics [21], and wavelength conversion efficiency [22]. Recently, the effects of dispersion fluctuations on the pump depletion of a PI-FOPA were also investigated, indicating that nanometer-scale fluctuations in the transverse geometry of a fiber can lead to qualitatively different saturation characteristics and nonreciprocal transmission characteristic [23]. To the best of our knowledge, the effects of longitudinal dispersion fluctuations on the pump depletion of a gain-saturated phase-sensitive amplifier (PSA) have not been investigated. In this paper, we theoretically investigate a fiber-based all-optical switch based on the pump depletion of a single-pump gain-saturated PSA, using an inhomogeneous fiber model. Moreover, nonreciprocal transmission characteristic of the PS all-optical switch induced by the ZDW fluctuations is analyzed.

\section{THEORY}

First, we will describe the operation principle of a parametric PS all-optical switch, compared to the PI counterpart. Then, an inhomogeneous fiber model including the ZDW fluctuations is put forward by a modified nonlinear Schrodinger equation (NLSE), in order to investigate the effects of ZDW fluctuations on the PS all-optical switch. As shown in Fig. 1(a), the PI all-optical switch is based on a single-pump gain-saturated PI-FOPA. In the PI-FOPA, the pump wave with high optical power $\left(\omega_{p}\right)$ and the signal wave with low optical power $\left(\omega_{s}\right)$ are injected into an HNLF. Through the FWM process, another wave called the idler is created at a new frequency $\omega_{i}$ given by $\omega_{i}=2 \omega_{p}-\omega_{s}$. At the output of the PI-FOPA, three waves with correlated phases are generated. The relative phase $\theta=\theta_{s}+\theta_{i}-2 \theta_{p}$, where $\theta_{s}, \theta_{i}$ and $\theta_{p}$ are the phases of the signal, idler and pump, respectively, determines the phase matching condition and thus the gain of the signal and idler. In a PI amplifier (PIA), the idler wave is generated irrespective of the initial relative phase, so that the PI gain only depends on the input signal power in the gain-saturated regime. Correspondingly, the pump depletion of the gain-saturated PIA changes with the input signal power, which is the principle of the PI optical switch. In contrast with the PI optical switch, the PS all-optical switch is based on a single-pump gain-saturated PS-FOPA. As shown in Fig. 1(b), the phase-correlated pump, signal and idler are generated through a cascaded PIA in the HNLF1 with the pump and signal waves at the input, which is called a copier stage [14]. A phase tuning unit is used to control the phases of the input signal and idler simultaneously by adding a modulation phase of $\theta \bmod$ [24], and correspondingly the relative phase among the three involved waves will change. As a result, by controlling the relative phase in a gain-saturated PSA, the power transferring from or to the pump can be manipulated, and this is the principle of the PS optical switch. Therefore, a high-power pump wave can be switched ON-OFF using a very weak signal/idler pair, by tuning the phases of the signal and idler waves in the PS optical switch rather than by tuning the intensity as is done in the PI counterpart. Please note that, the powers of the signal and idler waves are nearly equal in order to maximize the efficiency of the phase-sensitive process. Finally, after passing through a tunable optical bandpass filter (OBPF) centered at the pump wavelength, the pump is selected and measured. In a practical implementation, a phase-locked loop (PLL) is necessary to compensate the relative phase drifts among the three involved waves due to acoustic and thermal effects. The cascaded PIA process has been comprehensively investigated in the past few years. Therefore, we only model the PSA process in our simulation, as shown in the red box of Fig. 1(b). A three-wave model is commonly used to theoretically investigate a single-pump PSA, where two photons from the pump are transferred to the signal and idler waves [25]. The photon exchange process can be described by [26],

$$
\begin{aligned}
\frac{d P_{p}}{d z}= & -\alpha P_{p}-4 \gamma\left(P_{p}^{2} P_{s} P_{i}\right)^{1 / 2} \sin \theta \\
\frac{d P_{s}}{d z}= & -\alpha P_{s}+2 \gamma\left(P_{p}^{2} P_{s} P_{i}\right)^{1 / 2} \sin \theta \\
\frac{d P_{i}}{d z}= & -\alpha P_{i}+2 \gamma\left(P_{p}^{2} P_{s} P_{i}\right)^{1 / 2} \sin \theta \\
\frac{d \theta}{d z}= & \Delta \beta+\gamma\left(2 P_{p}-P_{s}-P_{i}\right) \\
& +\gamma\left[\left(P_{p}^{2} P_{s} / P_{i}\right)^{1 / 2}+\left(P_{p}^{2} P_{i} / P_{s}\right)^{1 / 2}\right. \\
& \left.-4\left(P_{s} P_{i}\right)^{1 / 2}\right] \cos \theta
\end{aligned}
$$

where $P_{p}, P_{s}$ and $P_{i}$ are the optical powers of the pump, signal and idler waves, $\alpha$ and $\gamma$ are the fiber attenuation coefficient and the nonlinear coefficient, and $\Delta \beta$ is the linear phase mismatch. The relative phase $\theta(z)$ is given by

$$
\theta(z)=\Delta \beta z+\theta_{s}(z)+\theta_{i}(z)-2 \theta_{p}(z)
$$

where $\theta_{s}, \theta_{i}$ and $\theta_{p}$ are the phases of the signal, idler and pump waves. The initial relative phase $\Delta \theta_{0}$ at the input of the PSA is written as $\theta_{s 0}+\theta_{i 0}-2 \theta_{p 0}$, where $\theta_{s 0}, \theta_{i 0}$ and $\theta_{p 0}$ are the initial phases of the signal, idler and pump waves, respectively. For the three-wave model, only the signal, idler and pump waves are considered, and any higher-order FWM products are neglected. However, for the gain-saturated FOPA, higher-order FWM products are inevitably generated. Furthermore, in order to investigate the effects of dispersion fluctuations on the PS pump depletion, a spatially varying dispersion has to be included. The variations of a HNLF core diameter result in random ZDW shifts, while the dispersion slope only varies weakly along the fiber, and can be approximated as a constant [27]. Consequently, parametric interactions in an inhomogeneous fiber model including the ZDW fluctuations can be described by standard nonlinear Schrodinger equation [28],

$$
\frac{d A}{d z}=\left(\hat{D}(z)+\hat{N}-\frac{\alpha}{2}\right) A
$$

where the dispersion operator $\hat{D}(z)$ has a spatial dependence to account for the ZDW fluctuations along the fiber, $\hat{N}$ is the nonlinear operator that governs the effect of fiber nonlinearities, 


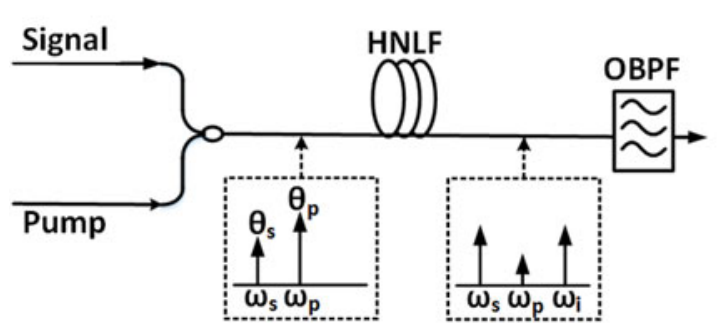

(a)

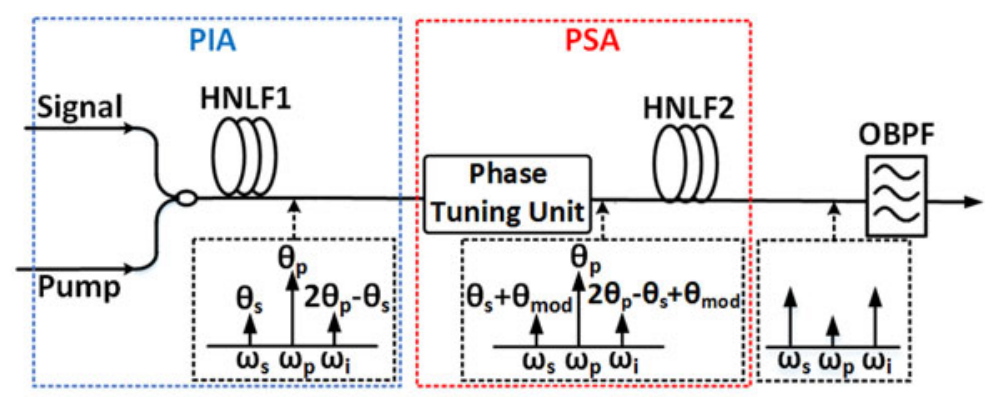

(b)

Fig. 1. General schemes of the parametric (a) PI, and (b) PS all-optical switch.

and $\alpha$ is the fiber attenuation coefficient. With a wide enough simulation bandwidth, all higher-order FWM products that contribute to pump depletion can be taken into account using the modified NLSE. Moreover, in a deeply gain-saturated FOPA, a significant part of the input pump power can be converted to the amplified vacuum noise, indicating that amplification of the vacuum noise may have a non-negligible effect on the pump depletion [23]. Therefore, the vacuum noise is modeled as a wideband Gaussian white noise with a power spectral density (PSD) of $h \nu_{p} / 2$. Meanwhile, we assume that the involved three waves co-propagate along the HNLF with the same polarization. The NLSE is numerically solved by dividing the fiber into 1000 segments with lengths $\Delta z$. The ZDW fluctuations along the fiber are modeled as correlated Gaussian noise with correlation length $L_{c}$ and standard deviation (STD) $\sigma_{\lambda_{0}}$. Under the condition that the segment length $\Delta z$ is much shorter than the nonlinear length $\left(\left(\gamma P_{p}\right)^{-1}\right)$, the ZDW can be treated as constant over the short fiber segment [19]. Thus in each fiber segment, the ZDW is assumed to be constant and defined as $\lambda_{0}\left(z_{k}\right)=\left\langle\lambda_{0}\right\rangle+\delta_{\lambda_{0}}\left(z_{k}\right)$, where $\left\langle\lambda_{0}\right\rangle$ is the global ZDW of the HNLF, and the local ZDW fluctuations $\delta_{\lambda_{0}}\left(z_{k}\right)$ are generated with the following equation [19], [29],

$$
\begin{aligned}
\delta_{\lambda_{0}}\left(z_{k}\right)= & \exp \left(-\frac{\Delta z}{L_{c}}\right) \delta_{\lambda_{0}}\left(z_{k-1}\right) \\
& +\sigma_{\lambda_{0}} \sqrt{1-\exp \left(-\frac{2 \Delta z}{L_{c}}\right)} \times r_{k}
\end{aligned}
$$

where $\Delta z=z_{k}-z_{k-1}(k=1,2 \ldots 1000), r_{k}$ has a Gaussian random distribution with the mean and STD of 0 and 1, respectively, and $\delta_{\lambda_{0}}\left(z_{0}\right)$ is generated according to the Gaussian random distribution with the mean of 0 and STD of $\sigma_{\lambda_{0}}$. In order to realize random ZDW profiles, an ensemble of local ZDW fluctuations $\delta_{\lambda_{0}}(z)$ are implemented with a pre-determined $\sigma_{\lambda_{0}}$ according to (7). Then, the actual STDs $\left(\sigma_{r}\right)$ of the generated $\delta_{\lambda_{0}}(z)$ are calculated and compared with the pre-determined $\sigma_{\lambda_{0}}$. Only the local ZDW fluctuations $\left(\delta_{\lambda_{0}}(z)\right)$, whose actual STDs $\left(\sigma_{r}\right)$ are nearly equal to the pre-determined $\sigma_{\lambda_{0}}$, are selected under the condition of $\left|\sigma_{r}-\sigma_{\lambda_{0}}\right|<0.001 \mathrm{~nm}$. For the selected $\delta_{\lambda_{0}}(z)$, the mean values are calculated and manually subtracted to ensure the mean values of $\delta_{\lambda_{0}}(z)$ zero. Next, the pre-determined global ZDW is added to $\delta_{\lambda_{0}}(z)$. Finally, random ZDW profiles with the pre-determined STD and global ZDW

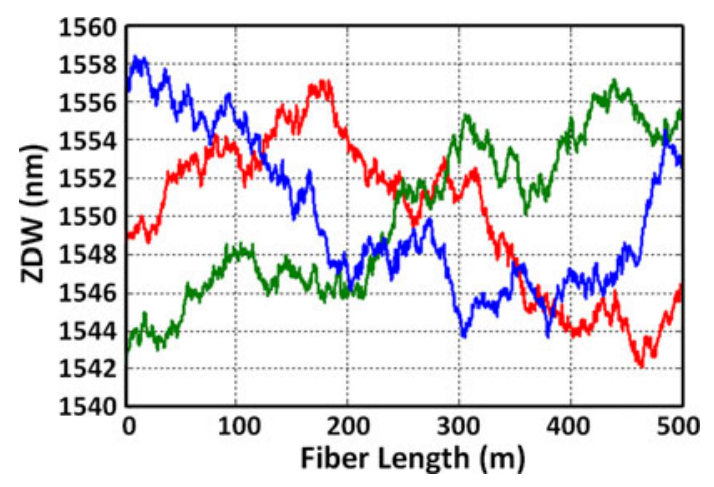

Fig. 2. Random ZDW profiles of three modeled inhomogeneous HNLFs.

are realized. Fig. 2 shows the random ZDW profiles of three modeled inhomogeneous HNLFs, with a length of $500 \mathrm{~m}$, a $\left\langle\lambda_{0}\right\rangle$ of $1550 \mathrm{~nm}$, a $\sigma_{\lambda_{0}}$ of $4 \mathrm{~nm}$, and a $L_{c}$ of $250 \mathrm{~m}$.

\section{EFFECTS OF DisPeRsion FluCtUATIONS ON A PS PARAMETRIC ALL-OPTICAL SWITCH}

In this section, we first assume a homogeneous HNLF and optimize powers and wavelengths of the signal and idler waves to maximize the pump depletion of a gain-saturated PSA, corresponding to the maximum extinction ratio (ER) of the PS all-optical switch using a homogeneous HNLF. The ER of the PS all-optical switch is defined as the ratio of the maximum and minimum output pump power, when the phases of the signal and idler waves are swept. Please note that the homogeneous HNLF here is a special case of our proposed inhomogeneous HNLF model under the condition of $\sigma_{\lambda_{0}}=0$, indicating that the HNLF is divided into several sections with the same ZDW. Then, based on these optimal signal/idler parameters, the ERs of the PS optical switches with inhomogeneous HNLFs are calculated and compared with that of the homogeneous HNLF based PS optical switch. Both the homogeneous and inhomogeneous HNLFs have the same global fiber parameters, including fiber length, loss, dispersion and nonlinearity coefficient. Through the comparison, effects of dispersion fluctuations along an inhomogeneous HNLF on the PS optical switch can be identified. In our simulation, we use an HNLF that is $500 \mathrm{~m}$ long, and has a global ZDW of $1550 \mathrm{~nm}$, a dispersion slope of $0.03 \mathrm{ps} / \mathrm{nm}^{2} / \mathrm{km}$, a nonlinear coefficient of $15 \mathrm{~W}^{-1} \mathrm{~km}^{-1}$, and an attenuation 


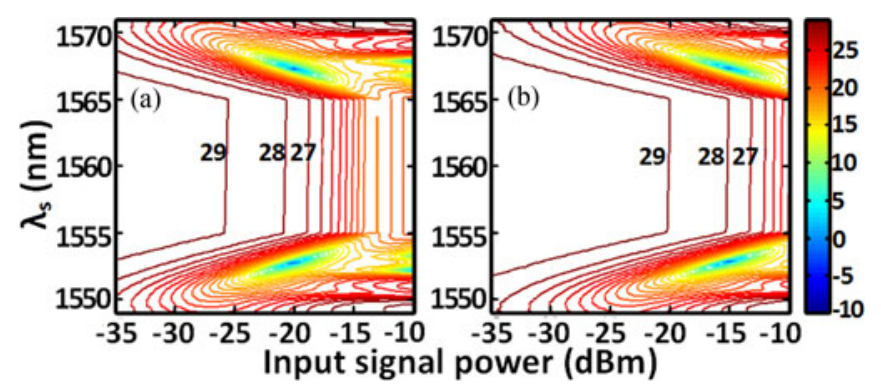

Fig. 3. Contour map of output pump power versus signal wavelength $\lambda_{s}$ and input power for (a) PSA, and (b) PIA. The color map represents the output pump power $(\mathrm{dBm})$ with a step of $1 \mathrm{~dB}$ in the contour.

coefficient of $0.7 \mathrm{~dB} / \mathrm{km}$. At the input of the HNLF, the pump power is set to $30 \mathrm{dBm}$ and its wavelength is $1560 \mathrm{~nm}$. The powers of the signal and idler waves are set to be equal to ensure the optimum phase-sensitive amplification [30]. Although the calculation can be repeated over many noise seeds, we use a prolonged simulation time window of $1.6 \mathrm{~ns}$ to increase the number of averaged samples and ensure the simulation accuracy [23]. The same seeded vacuum noise with power spectral density (PSD) of $h \nu_{p} / 2$ and bandwidth of $20.46 \mathrm{THz}$ are used for all the work in order to keep the simulation results repeatable, where $h$ and $\nu_{p}$ represent the Planck's constant and pump frequency, respectively.

\section{A. PS Parametric All-Optical Switch With a Homogeneous $H N L F$}

As shown in Fig. 3(a), using the given typical parameters of the HNLF and pump wave, the powers and wavelengths of the input signal and idler waves are optimized to maximize the pump depletion of the homogeneous HNLF based PSA. Here, the initial phases of three waves are set to 0 . The input signal and idler powers are set to be equal as in practical situation. The input signal (or idler) power varies from $-35 \mathrm{dBm}$ to -10 $\mathrm{dBm}$ with a step of $1 \mathrm{~dB}$ and signal wavelength varies from $1549 \mathrm{~nm}$ to $1555 \mathrm{~nm}$ and from $1565 \mathrm{~nm}$ to $1571 \mathrm{~nm}$ with a step of $0.1 \mathrm{~nm}$. The input signal/idler power accounts for the individual average power (not the sum of their individual powers) of the signal and idler injected into the PSA. For the purpose of comparison, pump depletion of the corresponding PIA is also optimized without any idler wave at the input as shown in Fig. 3(b). For a single-pump FOPA, only even-order dispersion parameters contribute to the linear phase mismatch, resulting in a symmetrical gain spectrum with respect to the pump wavelength. In a gain-saturated FOPA, along with the amplification of the signal and idler, the pump power is depleted. Thus, a symmetrical pump depletion is expected. This is applicable for both the PIA and PSA. As shown in Fig. 3, we find that the output pump power (pump depletion) is indeed well symmetrical with the respect to the spectral spacing between the signal and the pump waves. The minimum output pump power is obtained with a signal wavelength of either $1552.8 \mathrm{~nm}$ or $1567.3 \mathrm{~nm}$ for both the PSA and PIA, and with a lower input signal/idler power $(-20 \mathrm{dBm})$ for the PSA than the PIA $(-15 \mathrm{dBm})$. It is very

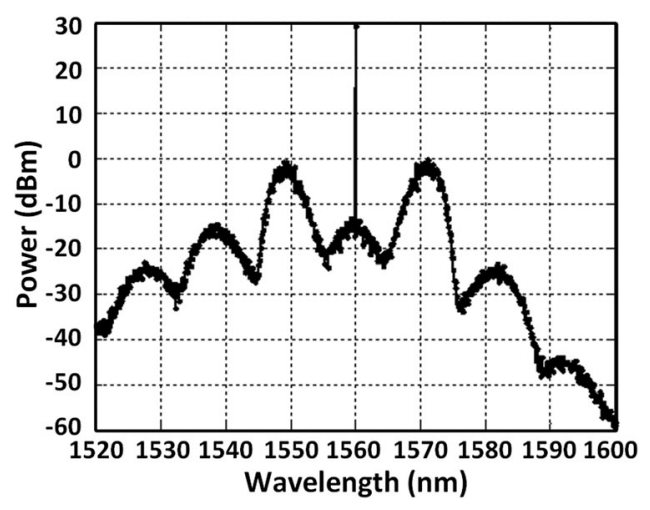

Fig. 4. Parametric fluorescence with input pump power of $30 \mathrm{dBm}$.

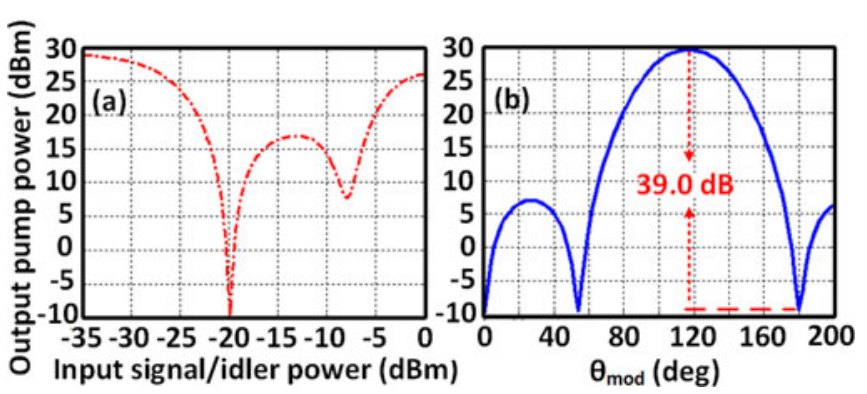

Fig. 5. Pump depletion as a function of (a) input signal/idler power, (b) phase variation of input signal/idler.

interesting that both PIA and PSA share the same optimal wavelength, due to the same phase matching condition. Here, we set the optimal signal wavelength at $1552.8 \mathrm{~nm}$. We also calculate the parametric fluorescence when only the pump is present at the HNLF input, as shown in Fig. 4. The output pump power is $29.4 \mathrm{dBm}$, indicating that the pump depletion induced by vacuum noise is only $0.25 \mathrm{~dB}$, in addition to the $0.35 \mathrm{~dB}$ fiber loss. As a result, the effect of parametric amplification of vacuum noise on pump depletion is very small, due to a relatively low nonlinear figure of merit (FoM) of 7.2 [23]. Besides, we find that the peak-gain wavelength is around $1550 \mathrm{~nm}$ and $1570 \mathrm{~nm}$. Therefore, the maximum pump depletion can be obtained, when the signal wavelength is set at approximately half wavelength spacing between the pump wavelength and the peak-gain wavelength, which matches the experimental observation [10].

Next, with the optimal signal wavelength of $1552.8 \mathrm{~nm}$, we calculate the pump depletion of the PSA by sweeping the input signal/idler power from $-35 \mathrm{dBm}$ to $0 \mathrm{dBm}$ in steps of $0.1 \mathrm{~dB}$, as shown in Fig. 5(a). A minimum output pump power of $-9.6 \mathrm{dBm}$, i.e. pump depletion of $39.0 \mathrm{~dB}$ is obtained under the condition that the input signal/idler power is set to be $-19.9 \mathrm{dBm}$. Thus, high PS pump depletion can be realized with a very low input signal/idler power. Based on the optimal signal wavelength of $1552.8 \mathrm{~nm}$ and input signal/idler power of $-19.9 \mathrm{dBm}$, we investigate the static response of the PS alloptical switch by tuning the phases of the signal and idler waves simultaneously from 0 to 200 degrees with a step of 2 degrees, as shown in Fig. 5(b). The ER of the PS all-optical switch is $39.0 \mathrm{~dB}$, indicating that it is possible to obtain a well-performing 


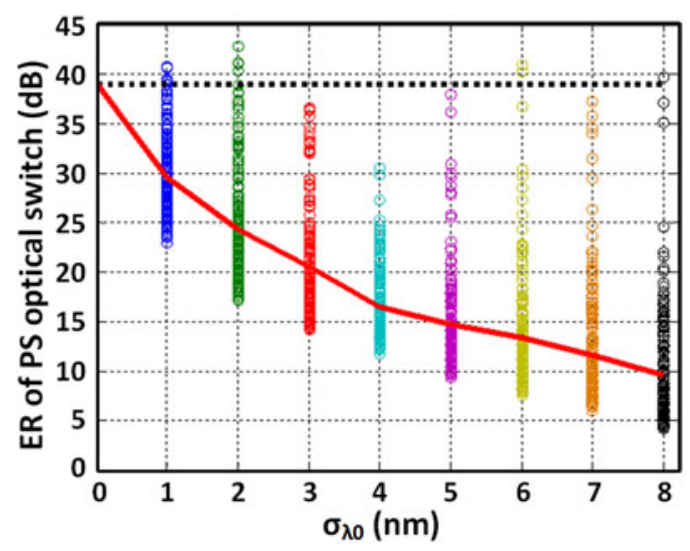

Fig. 6. ER of PS all-optical switch as a function of the ZDW standard deviations. Each circle represents the calculated ER of a PS all-optical switch from one member of the fiber ensemble with a specific ZDW profile. Identical global parameters are used. The black dotted line represents the ER of the homogeneous HNLF based PS optical switch, and the red line represents the average ER.

PS all-optical switch by dynamically tuning the phases of the signal and idler waves. Consequently, a guideline to optimize the PS all-optical switch can be outlined as follows: Firstly, the idler should be blocked at the input of the PS all-optical switch, and the input signal wavelength and power are optimized so that the pump depletion of the PIA is maximized. Secondly, based on the optimal signal wavelength and input power for the pump depletion of the PIA, high ER of PS all-optical switch can be obtained when the signal and idler powers are decreased simultaneously, because the optimal input signal/idler power of the PSA is lower than the optimal input signal power of the corresponding PIA.

\section{B. Effects of Dispersion Fluctuations}

By comparing the ER of the PS optical switch with a homogeneous and an inhomogeneous HNLF model, we start to explore the effects of dispersion fluctuations. We set the optimal signal and idler wavelengths to $1552.8 \mathrm{~nm}$ and $1567.3 \mathrm{~nm}$ respectively and the signal/idler power to $-19.9 \mathrm{dBm}$. Meanwhile, the ZDW is perturbed according to Eq. (7) with a correlation length $L_{c}$ of $250 \mathrm{~m}$. The STD $\sigma_{\lambda_{0}}$ is less than $8 \mathrm{~nm}$, due to the HNLF fabrication tolerance [31]. For each $\sigma_{\lambda_{0}}$, an ensemble of 200 pieces of HNLFs with stochastically random ZDW profiles are simulated. All HNLFs share the same global ZDW of $1550 \mathrm{~nm}$. As shown in Fig. 6, the red line represents the variations of the average ER of the PS all-optical switch versus the ZDW standard deviation, respectively. Each circle in the figure represents the calculated ER of a PS all-optical switch that consists of a member of the fiber ensemble. Please note that, in order to obtain the ER of each HNLF based PS optical switch (each circle in Fig. 6), except the individual ZDW profiles, all other global simulation parameters are identical. As above, we calculate the static responses of each PS optical switch by varying the signal/idler phases from 0 to 200 degrees in steps of 2 degrees. For a comparison, the ER of the corresponding homogeneous HNLF based PS all-optical switch is denoted as the horizontal black-dotted line. It can be
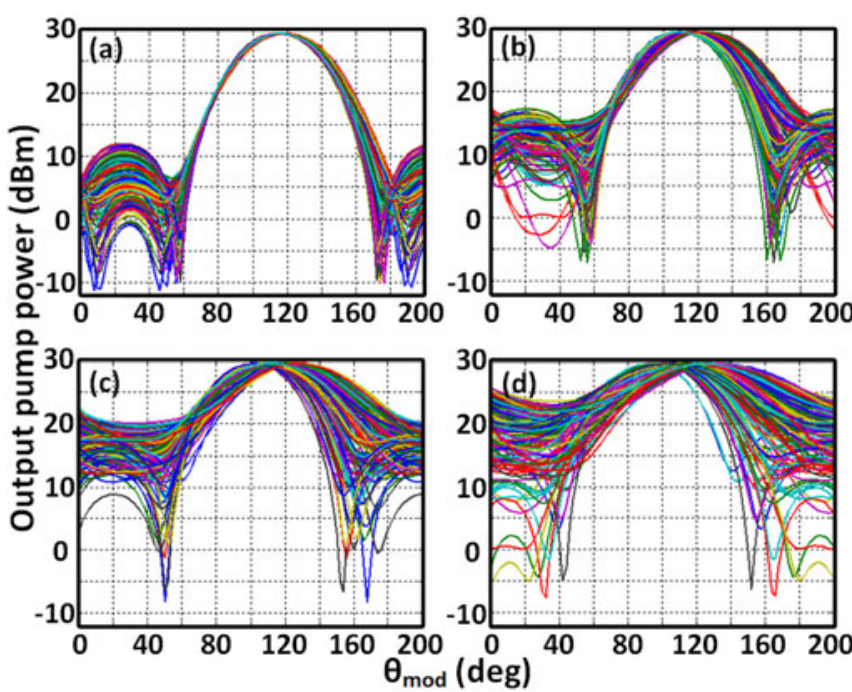

Fig. 7. Static responses of PS optical switches based on inhomogeneous HNLFs with different ZDW standard deviations of (a) $\sigma_{\lambda_{0}}=1 \mathrm{~nm}$, (b) $\sigma_{\lambda_{0}}=3 \mathrm{~nm}$, (c) $\sigma_{\lambda_{0}}=5 \mathrm{~nm}$, (d) $\sigma_{\lambda_{0}}=7 \mathrm{~nm}$. Each line represents the static response of a PS optical switch with a specific ZDW profile and STD.

observed that, with the increase of the STD $\sigma_{\lambda_{0}}$, the average ER of the PS all-optical switches is significantly decreased. Dispersion fluctuations with STD of only $1 \mathrm{~nm}$ will induce nearly $10 \mathrm{~dB}$ reduction of the average ER of the PS all-optical switches, compared with the corresponding homogeneous HNLF based PS optical switch. On the other hand, for some specific ZDW profiles, the ER of the PS optical switch can even exceed that of the homogeneous HNLF based counterpart. As shown in Fig. 6, more than $3 \mathrm{~dB}$ improvement of the ER can be achieved for a PS optical switch with designated dispersion fluctuations, because the locally controlled phase matching ensures a higher efficiency of power transfer from the pump to the signal/idler and highorder FWM products. Even though dispersion fluctuations are likely to deteriorate the performance of the PS optical switch under general conditions, it is possible to obtain a PS switch with a higher ER by controlling the local dispersion along the HNLF.

Next, the static responses of PS optical switches based on each HNLF ensemble with different ZDW profiles and STDs are shown in Fig. 7. For simplicity, here we just present the results under the conditions that $\sigma_{\lambda_{0}}$ is set to be $1 \mathrm{~nm}, 3 \mathrm{~nm}, 5 \mathrm{~nm}$ and $7 \mathrm{~nm}$, respectively. From Fig. 7 we see that, due to the dispersion fluctuations, the static responses of the PS optical switches vary significantly. When the STD of the ZDW is increased to $7 \mathrm{~nm}$, the ER can be reduced to nearly $5 \mathrm{~dB}$, as shown in Figs. 6 and 7(d), corresponding an ER decrease of about $34 \mathrm{~dB}$ in relevance to the homogeneous HNLF based PS optical switch with an ER of $39 \mathrm{~dB}$. Furthermore, we note that the dispersion fluctuations mainly affect the minimum (OFF-state) rather than the maximum (ON-state) PS output pump power, as the maximum pump power almost remains unchanged and the minimum pump power fluctuates severely, which can be explained from (1) to (4). The power transfer depends on the relative phase $\theta(z)$ among the three waves. The power can be transferred either from the pump to the signal and idler (when $\theta=\pi / 2$, parametric 
amplification and pump depletion) or from the signal and idler to the pump (when $\theta=-\pi / 2$, parametric de-amplification). From (5), by properly setting the initial signal and idler phases, the relative phase $\theta$ can be negative (i.e. $\theta<0$ ). Although longitudinal dispersion fluctuations result in random fluctuations of the local linear phase mismatch $\Delta \beta$, the pump power can't be transferred to the signal and idler waves due to the negative relative phase $\theta$. Therefore, with the proper initial signal and idler phases, the pump is not depleted at the presence of dispersion fluctuations, i.e. the maximum output pump powers of the ONstate almost keep unchanged for individual PS optical switches. On the other hand, at the OFF-state, the maximum pump depletion (minimum output pump power) is obtained. However, due to the longitudinal dispersion fluctuations, the local phase mismatch $\Delta \beta$ fluctuates randomly. From (4), the evolution of the relative phase $\theta(z)$ varies for PS optical switches based on HNLFs with different dispersion profiles. As a result, the output pump power of the OFF-state fluctuates seriously and the ERs of the PS all-optical switches are more likely to be reduced significantly. In addition, the peak power positions for the static responses of individual PS optical switches are deviated from each other with an increase of the dispersion fluctuations. The phase difference of $\theta_{\bmod }$ to obtain the maximum ER of the PS optical switch also changes, especially when $\sigma_{\lambda_{0}}$ is much larger. We infer that, with an increase of $\sigma_{\lambda_{0}}$, fluctuations of the linear phase mismatch $\Delta \beta$ increase correspondingly. From (4), the optimal initial relative phases $\Delta \theta_{0}$ corresponding to the maximum output pump power become different for the PS optical switches based on individual inhomogeneous HNLFs. Moreover, due to the random fluctuations at the OFF-state, the phase difference of $\theta_{\text {mod }}$ to obtain the maximum ER changes as well. Therefore, the dispersion fluctuations impose a severe challenge for the PS all-optical switch, and the local dispersion of HNLFs needs to be well controlled.

\section{NONRECIPROCAL TRANSMISSION CHARACTERISTIC}

It is known that a FOPA operating in the small-signal regime can exhibit the same gain, when the three involved waves are launched in the opposite direction, indicating that the inhomogeneous FOPA operating with negligible pump depletion is reciprocal [32]. However, the recent work shows that a FOPA operating in gain saturation is very sensitive to the local phase matching and becomes nonreciprocal due to the dispersion fluctuations along the HNLF [33]. Therefore, it is reasonable to consider the nonreciprocal transmission characteristic of the PS all-optical switch by reversing the input ports of the HNLF where gain saturation of the PSA occurs. For the simulation, we use a modeled inhomogeneous HNLF with a random ZDW profile denoted as the green line in Fig. 2. The $500 \mathrm{~m}$ HNLF has a global ZDW of $1550 \mathrm{~nm}$, a correlation length of $250 \mathrm{~m}$, a $\sigma_{\lambda_{0}}$ of $4 \mathrm{~nm}$, a dispersion slope of $0.03 \mathrm{ps} / \mathrm{nm}^{2} / \mathrm{km}$, a nonlinear coefficient of $15 \mathrm{~W}^{-1} \mathrm{~km}^{-1}$ and an attenuation coefficient of $0.7 \mathrm{~dB} / \mathrm{km}$. The pump power of $30 \mathrm{dBm}$ is set at the wavelength of $1560 \mathrm{~nm}$. The powers of the signal and idler waves are set equal for the PSA. Vacuum noise is accounted for, as in the above simulations.

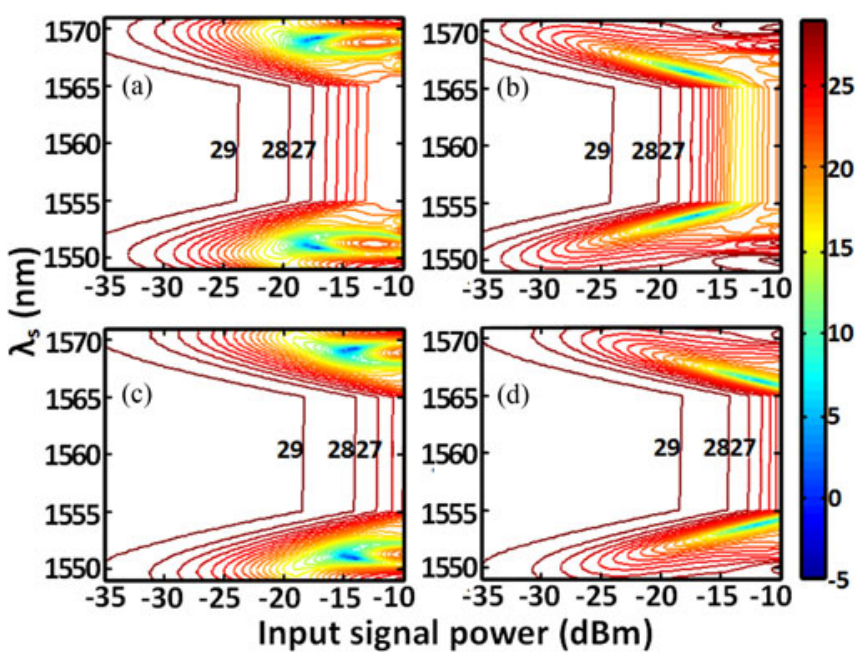

Fig. 8. Contour map of output pump power versus signal wavelength $\lambda_{s}$ and input power, (a) PSA in the forward direction, (b) PSA in the backward direction, (c) PIA in the forward direction, (d) PIA in the backward direction. The color map represents the output pump power $(\mathrm{dBm})$ with a step of $1 \mathrm{~dB}$ in the contour.

In Fig. 8, the pump depletion is shown as functions of the signal (idler) wavelength and input power for both the PSA and the PIA in two different propagation directions. Both the PSA and the PIA are basically reciprocal in the small-signal gain regime, but become nonreciprocal in the gain saturation regime. For the forward PSA in Fig. 8(a) and forward PIA in Fig. 8(c), the minimum output pump powers (corresponding to the maximum pump depletion) are $-14.1 \mathrm{dBm}$ and -14.0 $\mathrm{dBm}$, respectively, with a signal wavelength of $1550.9 \mathrm{~nm}$. However, for the backward PSA in Fig. 8(b) and backward PIA in Fig. 8(d), the minimum output pump powers are $-7.1 \mathrm{dBm}$ and $-7.0 \mathrm{dBm}$, respectively, with a signal wavelength of $1553.8 \mathrm{~nm}$. Furthermore, the minimum output pump power of the PSA is obtained with an input signal power of at least $3 \mathrm{~dB}$ lower than the PIA counterpart. Therefore, with a lower input signal/idler power, the nonreciprocal transmission characteristic of the PSA becomes more significant than that of the PIA. We believe this is due to the higher gain and twice (both signal and idler) as high total input powers of the PSA. Because of the non-reciprocity, for a practical PS all-optical switch operating under the optimal conditions, the transmission direction should not be reversed. Otherwise, the performance of the PS all-optical switch may be deteriorated, even though the optimal system parameters (signal/idler wavelength and power) for the opposite propagating direction are used. In order to further investigate the nonreciprocal transmission characteristic of the PS all-optical switch, static responses of the PS optical switch are calculated based on the three modeled inhomogeneous HNLFs with random ZDW profiles that are shown in Fig. 2. Other global parameters are identical as used in Fig. 8, except that the signal wavelength of $1552.8 \mathrm{~nm}$ and the input signal/idler power of $-19.9 \mathrm{dBm}$ are set. Vacuum noise is also included. By comparing Fig. 9(a) and (b), there are several differences between the forward and backward transmission. Firstly, the phase variations of the signal and idler $\left(\theta_{\bmod }\right)$ corresponding to the maximum and minimum pump power are different. Secondly, there is an obvious 


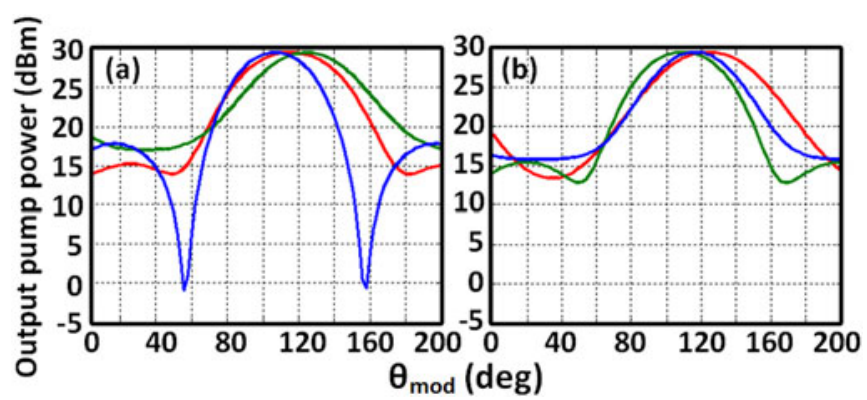

Fig. 9. Static responses of three PS optical switches for the (a) forward direction, (b) backward direction. The red, green and blue lines represent static responses of three PS optical switches using the inhomogeneous HNLFs denoted with the same colors as in Fig. 2.

difference at the minimum pump power, and thus different ERs of a PS all-optical switch are obtained for the forward and backward transmission due to the dispersion inhomogeneity. Thirdly, the curve shapes of the static responses are different. We infer that, due to different dispersion profiles for the forward and backward PS optical switch, local phase mismatch varies for two opposite directions. From (4), different evolutions of the relative phase $\theta$ are obtained, which result in different peak/valley positions and different pump depletion at the OFF-state for the two directions. Therefore, with transmission direction taken into consideration, the optimal phase difference of $\theta \bmod$ and the corresponding ER are different for a practical PS all-optical switch. Moreover, dispersion fluctuations have an effect on the power transfer from the input three waves (pump, signal and idler) to higher-order FWM products, which may further contribute to the different pump depletion of the OFF-state and the different static responses as a result of the different dispersion profiles of the two transmission directions.

\section{CONCLUSION}

We have theoretically investigated fiber-based parametric PS all-optical switch based on pump depletion of a gain-saturated single-pump PSA using an inhomogeneous fiber model. The effect of ZDW fluctuations on the PS all-optical switch has been analyzed. The minimum pump power fluctuated severely due to the dispersion fluctuation. Consequently, the ER of the PS all-optical switch is degraded to a large extent. In addition, due to the nonreciprocal transmission characteristic induced by the ZDW fluctuations, significantly different static responses of a PS all-optical switch can be obtained for the forward and backward propagation directions. Because of the fast response time of just a few femtoseconds of the FOPA, we believe that such PS optical switch has the potential of operating at very high speeds.

\section{REFERENCES}

[1] T. Torounidis, P. A. Andrekson, and B. E. Olsson, "Fiber-optical parametric amplifier with 70-dB gain," IEEE Photon. Technol. Lett., vol. 18, no. 10, pp. 1194-1196, May 2006.

[2] M. Jamshidifar, A. Vedadi, and M. E. Marhic, "Continuous-wave onepump fiber optical parametric amplifier with $270 \mathrm{~nm}$ gain bandwidth," in Proc. 2009 35th Eur. Conf. Opt. Commun., Vienna, Austria, 2009, Paper Mo.1.1.4.
[3] E. Ciaramella and S. Trillo, "All-optical signal reshaping via four-wave mixing in optical fibers," IEEE Photon. Technol. Lett., vol. 12, no. 7, pp. 849-851, Jul. 2000.

[4] R. Slavik et al., "All-optical phase and amplitude regenerator for nextgeneration telecommunications systems," Nature Photon., vol. 4, no. 10, pp. 690-695, 2010.

[5] G. W. Lu, T. Sakamoto, and T. Kawanishi, "Pump-phase-noise-tolerant wavelength multicasting for QAM signals using flexible coherent multicarrier pump," in Proc. Opt. Fiber Commun. Conf. Exhib., Los Angeles, CA, USA, 2015, Paper M2E.2.

[6] G. W. Lu, T. Sakamoto, and T. Kawanishi, "Coherently-pumped FWM in HNLF for 16QAM wavelength conversion free of phase noise from pumps," in Proc. 2014 Eur. Conf. Opt. Commun., Cannes, France, 2014, Paper P.1.16.

[7] Y. Meng, J. Lian, S. Fu, M. Tang, P. Shum, and D. Liu, "All-optical DPSK regenerative one-to-nine wavelength multicasting using dual-pump degenerate phase sensitive amplifier," J. Lightw. Technol., vol. 32, no. 15, pp. 2605-2612, Aug. 1, 2014.

[8] Z. Tong et al., "Towards ultrasensitive optical links enabled by low-noise phase-sensitive amplifiers," Nature Photon., vol. 5, no. 7, pp. 430-436, 2011.

[9] E. Temprana, V. Ataie, B. P. P. Kuo, E. Myslivets, N. Alic, and S. Radic, "Low-noise parametric frequency comb for continuous C-plus-L-band 16QAM channels generation,' Opt. Express, vol. 22, no. 6, pp. 6822-6828, 2014.

[10] S. Oda, H. Sunnerud, and P. A. Andrekson, "High efficiency and high output power fiber-optic parametric amplifier," Opt. Lett., vol. 32, no. 13, pp. 1776-1778, 2007.

[11] P. A. Andrekson, H. Sunnerud, S. Oda, T. Nishitani, and J. Yang, "Ultrafast, atto-Joule switch using fiber-optic parametric amplifier operated in saturation," Opt. Express, vol. 16, no. 15, pp. 10956-10961, 2008.

[12] A. Pejkic, R. R. Nissim, E. Myslivets, A. O. J. Wiberg, N. Alic, and S. Radic, "All-optical switching in a highly efficient parametric fiber mixer: Design study," Opt. Express, vol. 22, no. 19, pp. 23512-23527, 2014

[13] R. Nissim, A. Pejkic, E. Myslivets, B. P. Kuo, N. Alic, and S. Radic, "Ultrafast optical control by few photons in engineered fiber," Science, vol. 345, no. 6195, pp. 417-419, 2014.

[14] R. Tang, J. Lasri, P. S. Devgan, V. Grigoryan, P. Kumar, and M. Vasilyev, "Gain characteristics of a frequency nondegenerate phase-sensitive fiberoptic parametric amplifier with phase self-stabilized input," Opt. Express, vol. 13, no. 26, pp. 10483-10493, 2005.

[15] C. Lundström et al., "Experimental comparison of gain and saturation characteristics of a parametric amplifier in phase-sensitive and phaseinsensitive mode," in Proc. 2009 35th Eur. Conf. Opt. Commun., Vienna, Austria, 2009, Paper 1.1.1.

[16] J. Kakande et al., "Detailed characterization of a fiber-optic parametric amplifier in phase-sensitive and phase-insensitive operation," Opt. Express, vol. 18, no. 5, pp. 4130-4137, 2010.

[17] J. Parra-Cetina, A. Kumpera, M. Karlsson, and P. A. Andrekson, "Phasesensitive fiber-based parametric all-optical switch," Opt. Express, vol. 23, no. 26, pp. 33426-33436, 2015.

[18] M. Karlsson, "Four-wave mixing in fibers with randomly varying zerodispersion wavelength," J. Opt. Soc. Amer. B, vol. 15, no. 8, pp. 2269-2275, 1998.

[19] M. Farahmand and M. de Sterke, "Parametric amplification in presence of dispersion fluctuations," Opt. Express, vol. 12, no. 1, pp. 136-142, 2004.

[20] F. Yaman, Q. Lin, S. Radic, and G. P. Agrawal, "Impact of dispersion fluctuations on dual-pump fiber-optic parametric amplifiers," IEEE Photon. Technol. Lett., vol. 16, no. 5, pp. 1292-1294, May 2004.

[21] P. Velanas, A. Bogris, and D. Syvridis, "Impact of dispersion fluctuations on the noise properties of fiber optic parametric amplifiers," J. Lightw. Technol., vol. 24, no. 5, pp. 2171-2178, May 2006.

[22] J. S. Y. Chen, S. G. Murdoch, R. Leonhardt, and J. D. Harvey, "Effect of dispersion fluctuations on widely tunable optical parametric amplification in photonic crystal fibers," Opt. Express, vol. 14, no. 20, pp. 9491-9501, 2006.

[23] R. R. Nissim, E. Myslivets, and S. Radic, "Performance limits of inhomogeneous fiber optic parametric amplifiers operated in saturated regime," $J$. Lightw. Technol., vol. 32, no. 21, pp. 3552-3559, Nov. 2014.

[24] C. Lundström, B. J. Puttnam, Z. Tong, M. Karlsson, and P. A. Andrekson, "Experimental characterization of the phase squeezing properties of a phase-sensitive parametric amplifier in non-degenerate idler configuration," in Proc. 36th Eur. Conf. Exhib. Opt. Commun., Torino, Italy, 2010, Paper Th.10.C.1. 
[25] C. Lundström, B. Corcoran, M. Karlsson, and P. A. Andrekson, "Phase and amplitude characteristics of a phase-sensitive amplifier operating in gain saturation," Opt. Express, vol. 20, no. 19, pp. 21400-21412, 2012.

[26] G. Cappellini and S. Trillo, "Third-order three-wave mixing in singlemode fibers: Exact solutions and spatial instability effects," J. Opt. Soc. Amer. B., vol. 8, no. 4, pp. 824-838, 1991.

[27] E. Myslivets, N. Alic, J. Windmiller, and S. Radic, "A new class of high-resolution measurements of arbitrary-dispersion fibers: Localization of four-photon mixing process," J. Lightw. Technol., vol. 27, no. 3, pp. 364-375, Feb. 2009.

[28] G. P. Agrawal, Nonlinear Fiber Optics, 5th ed. San Diego, CA, USA: Academic, 2013

[29] J. M. Chavez Boggio, J. D. Marconi, and H. L. Fragnito, "Double-pumped fiber optical parametric amplifier with flat gain over $47-n m$ bandwidth using a conventional dispersion-shifted fiber," IEEE Photon. Technol. Lett., vol. 17, no. 9, pp. 1842-1844, Sep. 2005.

[30] M. Vasilyev, "Distributed phase-sensitive amplification," Opt. Express, vol. 13, no. 19, pp. 7563-7571, 2005.

[31] M. Hirano, T. Nakanishi, T. Okuno, and M. Onishi, "Silica-based highly nonlinear fibers and their application," IEEE J. Sel. Topics Quantum Electron. vol. 15, no. 1, pp. 103-113, Jan. 2009.

[32] M. E. Marhic, G. Kalogerakis, and L. G. Kazovsky, "Gain reciprocity in fibre optical parametric amplifiers," Electron. Lett., vol. 42, no. 9, pp. 519-520, 2006.

[33] R. R. Nissim, A. Pejkic, E. Myslivets, N. Alic, and S. Radic, "Origin of non-reciprocal response in fiber optic parametric amplifiers," J. Lightw. Technol., vol. 33, no. 2, pp. 495-502, Jan. 2015. 